\title{
Function of 14-3-3 proteins
}

SIR - 14-3-3 proteins were first characterized as very abundant acidic proteins in the brain ${ }^{1}$, functioning as kinasedependent activators of tyrosine and tryptophan hydroxylase involved in the biosynthesis of neurotransmitters ${ }^{2}$. More recent studies indicate that they are multifunctional regulators participating in cell-cycle control and various signal transduction pathways ${ }^{3,4}$. Their functions are, however, still incompletely
In the process of characterizing the human $14-3-3 \varepsilon$ cDNA, we found unexpectedly that the $3^{\prime}$ untranslated region of this cDNA showed a $285 \mathrm{bp}$ match with clone 8-1 of the probe for Miller-Dieker lissencephaly syndrome $^{6,7}$ (see figure). Because the 8-1 sequence aligned with the 3 ' untranslated region of the human $14-3-3 \varepsilon$ cDNA, we assessed the validity of this match by comparing the human sequence to corre-

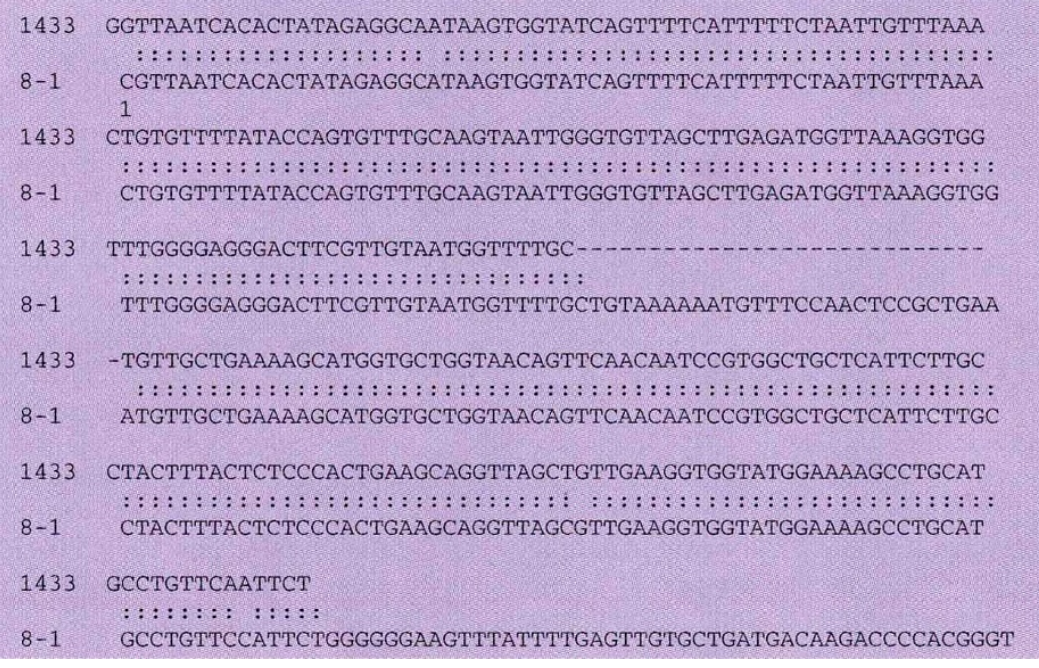

Sequence alignment of the human 14-3-3 $\varepsilon$ and MDS 8-1 probe. Dots indicate identity. Bars indicate gap. GenBank accession numbers of the aligned sequences: human 14-3-3E , U43399; MDS 8-1 probe, L13388. The presented alignment starts at nucleotide 1204 of human 14-3-3ع.

\section{understood.}

We have been studying the molecular mechanisms by which $14-3-3$ proteins interact with cellular and viral oncoproteins. In this process, we independently isolated a 1,706 base-pair (bp) human 143-3 $\varepsilon$ complementary DNA containing an intact $3^{\prime}$ untranslated region with a poly $(\mathrm{A})$ tail (GenBank accession number U43399). The $14-3-3 \varepsilon$ protein sequence translated from this cDNA is identical to human $14-3-3 \varepsilon$ sequence recently deposited in the database ${ }^{5}$, for which the $3^{\prime}$ untranslated region was not reported.

\footnotetext{
1. Aitken, A. Trends Biochem. Sci. 17, 498-501 (1992).

2. Ichimura, T. et al. Proc. Natl Acad. Sci. USA. 85 7084-7088 (1988)

3. Burbelo, P. D. \& Hall, A. Curr. Biol. 5, 95-96 (1995)

4. Morrison, D. Science 266, 56-57 (1994).

5. Conklin, D. S., Galaktionov, K., Beach, D. Proc. Natl Acad. Sci. U.S.A. 92, 7892-7896 (1995).

6. Reiner, O. et al. Nature 364, 717-721 (1993).

7. Dobyns, W. B. \& Truwit, C. L. Neuropediatrics 26,132-147 (1995).

8. Kozak, C. et al. Mammalian Genome 6, 142-144 (1995).

9. Kurtz, A. \& Zimmer, A. Mammalian Genome 6, 145-146 (1995)

10. Selivanov, N. A., Prilipov, A. G., Efimov, V. P., Marusich, E. I. \& Mesyanzhinov, V. V. Biomed. Sci. 1, 55-62 (1990).

11. Yanicostas, C. \& Lepesant, J. -A. Mol. gen. Genet. 224 450-458 (1990)

12. Shayiq, R. M. \& Avdhani, N. G. J. Biol. Chem. 267 2421-2428 (1992)

13. Tetsu, O.,Kanno, R., Isono, K., Taniguchi, M. \& Kanno, M. Biochim. Biophys. Acta 1305, 109-112 (1996). 14. Reiner, O., et al. J. Neurosci. 15, 3730-3738 (1995).
}

sponding regions in the mouse and rat $14-3-3 \varepsilon$ homologues. We found perfect alignment throughout the length of the three 14-3-3 sequences (including the 3' distal untranslated regions: human 14-3$3 \varepsilon$, U43399; mouse $14-3-3 \varepsilon$, Z19599; and rat $14-3-3 \varepsilon, D 30739$ ), strongly suggesting that the 3 ' untranslated region of the 14$3-3 \varepsilon$ gene is highly conserved.

This sequence information suggests that 14-3-3 gene maps close to or within the previously defined 8-1 Miller-Dieker lissencephaly probe. To confirm directly the map location of the $14-3-3 \varepsilon$ gene in the mammalian genome, we used as a probe a 3 ' cDNA fragment unique to the human $14-3-3 \varepsilon$ and divergent from other 14-3-3 subtypes to type the progeny of an interspecies Mus spretus genetic cross ${ }^{8}$. We typed progeny for a Hind III restriction-fragment length polymorphism of 14-3-3ع which shows tight linkage to Evi2. We. could not identify any recombinants for this gene in 88 progeny, indicating that, at the upper level of the $95 \%$ confidence level, these genes are separated by $0-3.3$ centimorgan. This position is in good agreement with a previous study which positioned the mouse homologue of Miller-Dieker chromosomal region (MDCR) just proximal to Evi2 ${ }^{9}$.

Based on our results, we conclude that the human 14-3-3 gene is probably contained in the 8-1 probe previously used to define the $5^{\prime}$ end of the MDCR locus. Hence, the 8-1 probe is chimaeric for more than one gene, and the exact boundaries of the deletions associated with Miller-Dieker lissencephaly syndrome require further clarification.

Dong-Yan Jin

Myung Soo Lyu

Christine A. Kozak

Kuan-Teh Jeang*

Laboratory of Molecular Microbiology,

National Institute of Allergy and Infectious Diseases,

National Institutes of Health,

Bethesda, Maryland 20892-0460, USA

* To whom correspondence should be addressed. e-mail: kjeang@atlas.niaid.gov

REINER AND CASKEY REPLY - We agree with the mapping data presented by Jeang et al. above, but disagree with the interpretation. Our results indicate that the complementary DNA 8-1 encoding LIS-1 (ref. 6) overlaps the $3^{\prime}$ end of the 14-3-3ع gene. Two methods support this finding: first, we isolated cDNA 8-1 from a human fetal brain library; and second, we have demonstrated the existence of this transcript in RNA from both human fetal brain and mouse fetal brain by reverse transcriptase-polymerase chain reaction ( $R T-P C R$ ).

Because of the low expression level of the 14-3-3 transcript (tested by Northern blot analysis) and the existence of at least five LIS-1 transcripts, we used RT-PCR as a sensitive detection tool to investigate this gene product. As the presence of this rare transcript in more than one species may emphasize its importance, we amplified both human fetal brain RNA and mouse brain RNA. We found that the 14-3-3 LIS-1 overlapping transcript is detectable both in human and mouse (figure and experimental details available from $\mathrm{O}$. $\mathrm{R}$. on request).

Our initial genomic analysis of a human cosmid clone indicates that the $3^{\prime}$ sequences derived from 14-3-3 are located in one exon. We are now examining these genes in the Fugu fish genome, which has the advantages of a high degree of conservation, extensive synteny and a compact size. Overlapping genes are a well-known phenomenon among viruses ${ }^{10}$, but have rarely been described in vertebrates and invertebrates (see refs 11-13 for some examples). As LIS-1 is a gene tightly controlled during development $^{14}$, we are presently investigating the regulation of transcription of the 14-3-3LIS-1 mRNA during development.

\section{Orly Reiner}

Department of Molecular Genetics, The Weizmann Institute of Science,

76100 Rehovot, Israel

C. T. Caskey

Merck Reasearch Laboratories

RY 80K Rahway, 0765 New Jersey, USA 